\title{
Erratum to: RASSF1A, APC, ESR1, ABCB1 and HOXC9, but not p16INK4A, DAPK1, PTEN and MT1G genes were frequently methylated in the stage I non-small cell lung cancer in China
}

\author{
Qiang Lin · Junfeng Geng $\cdot$ Kelong Ma $\cdot$ Jian Yu $\cdot$ Jinfeng Sun \\ Zhenya Shen $\cdot$ Guoliang Bao $\cdot$ Yinming Chen $\cdot$ Hongyu Zhang \\ Yinghua He $\cdot$ Xiaoying Luo $\cdot$ Xu Feng $\cdot$ Jingde Zhu
}

Published online: 9 October 2009

(C) Springer-Verlag 2009

Erratum to: J Cancer Res Clin Oncol (2009)

135:1675-1684

DOI 10.1007/s00432-009-0614-4

The affiliation of Qiang Lin in the article, titled "RASSF1A, APC, ESR1, ABCB1 and HOXC9, but not p16INK4A, DAPK1, PTEN and MT1G genes were

The online version of the original article can be found under doi:10.1007/s00432-009-0614-4.

Q. Lin · J. Geng

Department of General Thoracic Surgery,

Shanghai Chest Hospital, Shanghai Jiaotong University,

200030 Shanghai, China

K. Ma $\cdot$ J. Yu $\cdot$ J. Sun $\cdot$ Y. Chen $\cdot$ H. Zhang $\cdot$ Y. He $\cdot$ X. Luo *

J. Zhu ( $\square)$

Cancer Epigenetics and Gene Therapy Program,

The State-Key Laboratory for Oncogenes and Related Genes,

Shanghai Cancer Institute, Shanghai Jiaotong University,

LN 2200/25, Xietu Road, 200032 Shanghai, China

e-mail: zhujingde@gmail.com

URL: http://www.shsci.org/eyjz3.htm

Q. Lin . Z. Shen

The First Hospital Affiliated to Suzhou University,

215006 Suzhou, Jiangsu, China

G. Bao

Division of Basic Research, Shanghai Chest Hospital,

Shanghai Jiaotong University, 200030 Shanghai, China

X. Feng

Department of Urology, Tumor Hospital,

Guangxi Medical University, 530022 Nanning, China

J. Zhu

Cancer Epigenetics Laboratory,

Obstetrics and Gynecology Hospital,

Fudan University, 200011 Shanghai, China frequently methylated in the stage I non-small cell lung cancer in China" DOI 10.1007/s00432-009-0614-4 should be "The First Hospital Affiliated to Suzhou University, 215006 Suzhou, Jiangsu, China" and "Department of General Thoracic Surgery, Shanghai Chest Hospital, Shanghai Jiaotong University, 200030 Shanghai, China". 